\title{
Combination of Different Techniques in Cryo-Electron Tomography with a Volta Phase Plate
}

\author{
Maryam Khoshouei ${ }^{1}$, Günther Gerisch ${ }^{2}$, Maria Ecke ${ }^{2}$, Julio Ortiz ${ }^{1}$, Jürgen M. Plitzko ${ }^{1}$, Radostin Danev ${ }^{1}$, \\ Wolfgang Baumeister ${ }^{1}$
}

1. Department of Molecular Structural Biology, Max-Planck Institute for Biochemistry, Am Klopferspitz 18, D-82152, Martinsried, Germany.

2. Department of Cell Dynamics, Max-Planck Institute for Biochemistry, Am Klopferspitz 18, D-82152, Martinsried, Germany.

Due to the sensitivity of the biological samples to beam irradiation resulting from induced changes to their structure, the limited allowed dose to frozen-hydrated biological specimens leads cryo images typically noisy and low in contrast. One way to improve the contrast is by applying few microns of defocus which is a well-known conventional technique since many years ago. The drawback of this technique is resolution deterioration caused by applied defocus that suppresses high-frequency information due to its sine type CTF. As opposed to the convectional technique, another way of increasing contrast is using a phase plate. Phase plate is located on the back-focal plane of the TEM. It changes the sine type CTF to a cosine type and the object information in the low spatial frequency domain is transferred well into the image information. There are various types of phase plates such as electrostatic, magnetic, anamorphotic, tulip type and etc. Nevertheless, none of them is as applicable as the thin film phase plate in terms of difficulties in fabrication and usage [1]. Among all different types of thin film phase plate, Zenrike phase plate has been the most successful one until the proposed novel phase plate. In the last two years, Volta phase plate (VPP) was developed and it improves on the performance of the Zernike phase plate by having a longer life time, overall better performance, simplicity in use and no fringe artefacts. The performance of the VPP is based on volta potential resulting from the incident electron beam and physical and chemical changes on the surface of the carbon [2].

Moreover, one of the model systems to evaluate of the performance of the VPP is an earth worm sperm. The worm sperm was obtained from the seminal receptacles bags of the Lumbricus terretris. Understanding the morphological and ultrastructural information play a significant role in taxonomic and phylogenetic studies. However, this study could not be done unless applying the combination of cryo-electron tomography (CET) techniques and VPP.

This huge cell comprises different compartments such as acrosome, nucleus, midpiece and flagellum. In this work, the combination of batch CET, single axis CET and dual axis CET with VPP are shown to observe different segments of this specimen. This is a first time to observe such a complicated vitrified biological system with a preserved structure and more importantly in the physiological status. In contrast, from the pertinent researches this sample was investigated based on tissue fixation and plastic sectioning with less visible structural details [3]. In the current work, the improved contrast makes the structural details more visible. These structural details could be critical factors in the cell motility, signaling of the sperm cell towards an ovum and fertilization process. 
[1] R Danev et al, Journal of Structural Biology 171 (2010), p. 174.

[2] R Danev et al, Proceedings of the National Academy of Sciences of the United States of America (2014).

[3] B. G. M Jamieson, Journal of Zoology 26 (1978), p. 225.
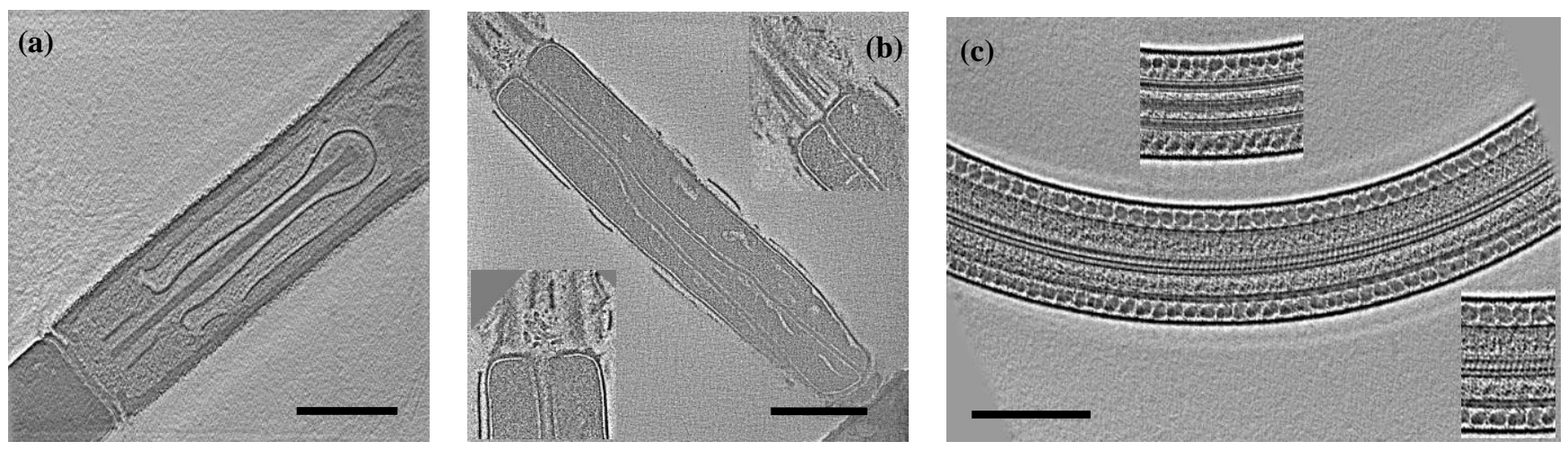

Figure 1. Slices of CET with the VPP (a) Single axis CET with the VPP from the end of the acrosome, scale bar: 400nm (b) Dual axis CET with the VPP from the midpiece. Visibility of a transition gate (left inset image) and triplet microtubules (right inset image), scale bar: 400nm. (c) Single axis CET with the VPP from the flagellum. Visibility of the doublet microtubules (upper inset image) and two singlets (lower inset image), scale bar: 400nm. [Titan Krios 300kV, energy filter, direct detector, defocus: 500nm, Magnification: 33000, 26000; Pixel size: 0.421 and $0.531 \mathrm{~nm}$ respectively].

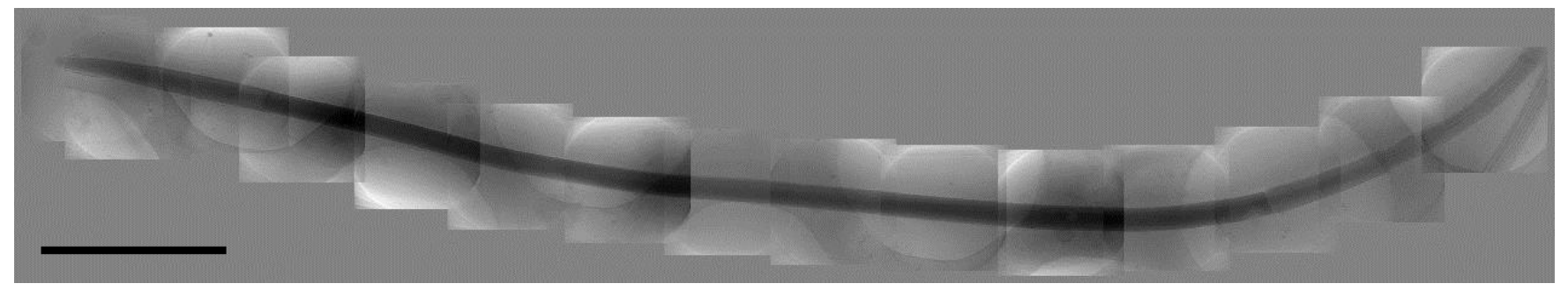

Figure 2. One projection of batch CET with the VPP. 15 single axis CET with a complete overlap from a worm sperm cell Slices of CET with the VPP. [Titan Krios 300kV, energy filter, direct detector, defocus: -500nm, Magnification: 19500, Pixel size: $0.71 \mathrm{~nm}$ ]. Scale bar: $3 \mu \mathrm{m}$ 